\title{
Mechanism and Improvement of Charged-Particles Transition in Microcup Electrophoretic Displays
}

\author{
Yi-Pai Huang, Fang-Cheng Lin, Szu-I Wu, and Po-Ru Yang
}

\begin{abstract}
Electrophoretic display (EPD) is one of the most prominent technologies used in electronic paper. EPDs have good paper-like readability in ambient sunlight, and their bistability characteristics allow them to use very little power. To understand the physical mechanism at work in EPDs in terms of optical phenomena and current responses, we investigated EPD charging behavior using a transient current, an intermediate-state current, a discharge current, and a current bump. After establishing the microcup EPD model, we developed recommendations for a shortened transition time. As our experiments show, the method can reduce the transition time by $20 \%$.
\end{abstract}

Index Terms-Microcup electrophoretic displays (EPDs), physical mechanism, current bump, current response, optical response.

\section{INTRODUCTION}

$\mathbf{L}$ IQUID CRYSTAL displays (LCDs) are widely used in consumer electronics because they possess several advantages, including a high frame rate sufficient for playing videos, high brightness, high contrast, high color saturation, and lightweight [1]-[3]. However, LCDs suffer from glare under sunlight, a narrow viewing angle, and low reflectance. Additionally, LCDs consume more power and are less stress-resistant in comparison to electrophoretic displays (EPDs). For these reasons, EPDs have become popular in electronic paper (e-paper) applications.

Research on EPDs has largely focused on manufacturing [4], image stability [5], [6], and image quality [7]. Bert and Smet described the dissociation of surfactants, as shown in Fig. 1(a) [8]. Yoshio and Tetsuo studied the formation of charged species during electrophoretic deposition, which enables pigments to become positively or negatively charged according to the functional groups [9]. Croucher and Hair described how surfactants provide long-term colloidal stability to the dispersion [10]. Bert and Smet described how the formation of micelles stabilizes the charged species in a non-polar solvent [11]. Upon being dissolved in solvent, surfactants dissociate themselves. Some of

Manuscript received August 30, 2012; revised February 07, 2013 and February 26, 2013; accepted February 26, 2013. Date of publication March 27, 2013; date of current version August 07, 2013. This work was supported in part by the National Science Council in Taiwan for Academic Projects NSC 99-2221-E-009-065-MY3 and NSC 99-2221-E-009-066-MY3.

Y.-P. Huang and F.-C. Lin are with the Department of Photonics \& Display Institute, National Chiao Tung University, Hsinchu, 30010, Taiwan (e-mail: fclin.eo93g@nctu.edu.tw).

S.-I Wu is with the Department of Photonics \& Institute of Electro-Optical Engineering, National Chiao Tung University, Hsinchu, 30010, Taiwan.

P.-R. Yang is with SiPix Technology, Inc., 33383 New Taipei City, Taiwan.

Color versions of one or more of the figures are available online at http:// ieeexplore.ieee.org.

Digital Object Identifier 10.1109/JDT.2013.2251859

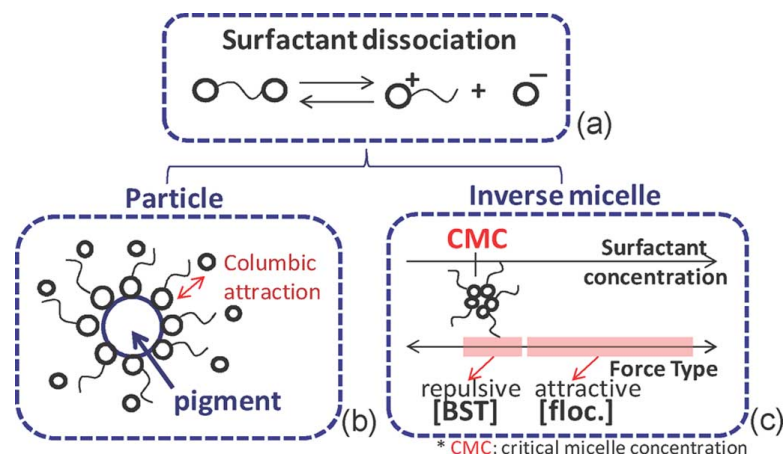

Fig. 1. The configuration of charged species inside a microcup EPD. (a) Dissociation of surfactants. (b) The positively charged pigment. (c) The forces acting on inverse micelles under varying surfactant concentrations.

the dissociated ions adhere to the surface of pigments and become charged dispersed particles, as shown in Fig. 1(b), while others form micelles, as shown in Fig. 1(c). The charged shells help to prevent flocculation of dispersed particles, which thus increases the dispersion stability [12]. However, the presence of too many unadsorbed ions would compress the electric double layers [13], which would negatively affect the dispersiveness of the suspensions.

Bert and Smet performed several studies of current response from 2003 to 2006 involving both dispersed particles and micelles in e-paper [14]-[16]. Roberts demonstrated how reverse micelles affect the electrostatic charging of non-polar colloids [17], and Chen described particle charge, mobility, and zeta potential in non-polar colloids [18]. However, our understanding of the physical mechanism of an EPD is incomplete. Few studies have been performed to examine internal charge behaviors. While applying an external electrical field, both charged particles and charged micelles would contribute to a transient current in an EPD device. However, only the motion of charged particles would change the optical states (reflectance). As a result, by synchronizing the optical response with the current behaviors, the motions of charged particles and charged micelles can be distinguished.

The optical contrast of an EPD is varied by manipulating the positions of electrically charged particles inside the solvent. For instance, a microcup EPD reveals a white state, while white particles with positive charges are attracted to the upper oppositely charged electrode. The microcup EPD reveals a black state when particles are repelled from the upper charged electrode. Light is absorbed by the black fluid inside a microcup EPD. However, the dispersed particles are not the only contributors to current that make the system more complex and difficult to control. This work aims to describe the physical mechanism 


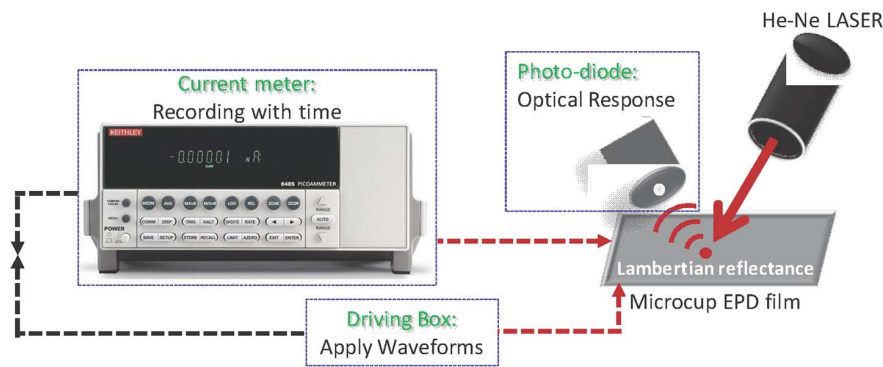

Fig. 2. The experimental setup for measuring current and optical responses under different driving waveforms.

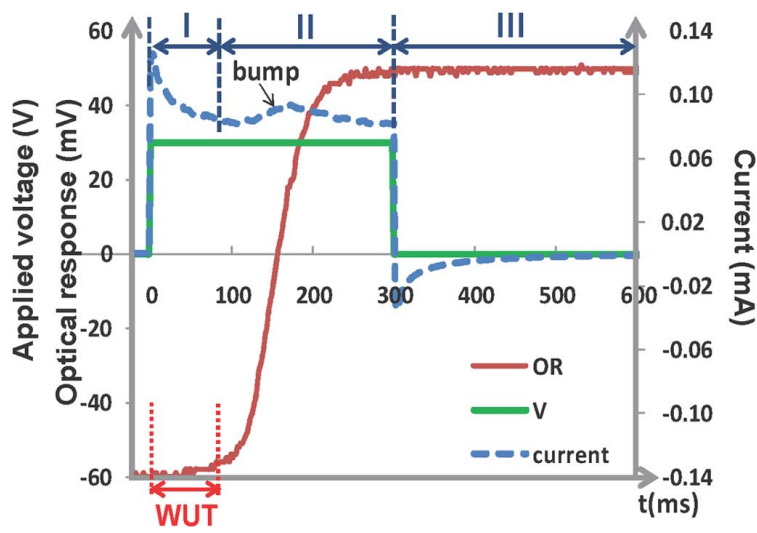

Fig. 3. Current and optical responses to a $30 \mathrm{~V}, 300 \mathrm{~ms}$ pulse voltage. The green square line is the applied voltage $(\mathrm{V})$; the blue dashed and red solid curves are the current and optical responses (OR), respectively.

of particle transition inside an EPD in terms of optical and current behaviors. Furthermore, with an understanding of the behavior of particles, we propose a driving method to reduce the transition time for practical applications.

\section{Testing the Physical Mechanism of Particle TRANSITION}

\section{A. Setup}

Both current and optical responses are analyzed to understand the physical mechanism of particle transition in this paper. The experimental setup is shown in Fig. 2. The light source was a $\mathrm{He}-\mathrm{Ne}$ laser with a wavelength of $632.8 \mathrm{~nm}$. The gray level (lightness) was modulated by controlling the input of electric waveforms from the driving box. The Lambertian reflected light was captured by a photo-diode to quantify gray levels. Simultaneously, the current response was acquired by a current meter.

\section{B. Current and Optical Responses in an EPD}

When a pulse voltage of $30 \mathrm{~V}$ with a $300 \mathrm{~ms}$ duration is applied, the EPD does not show an immediate optical response. The time from the application of voltage to a $10 \%$ variation in optical response is defined as the wake-up time (WUT). This duration is interpreted as the time required for differences in lightness to be perceived.

Based on the dominant contributors from particles, micelles or dissociated ions, the current response was divided into three parts, including a transient current (stage I), an intermediate

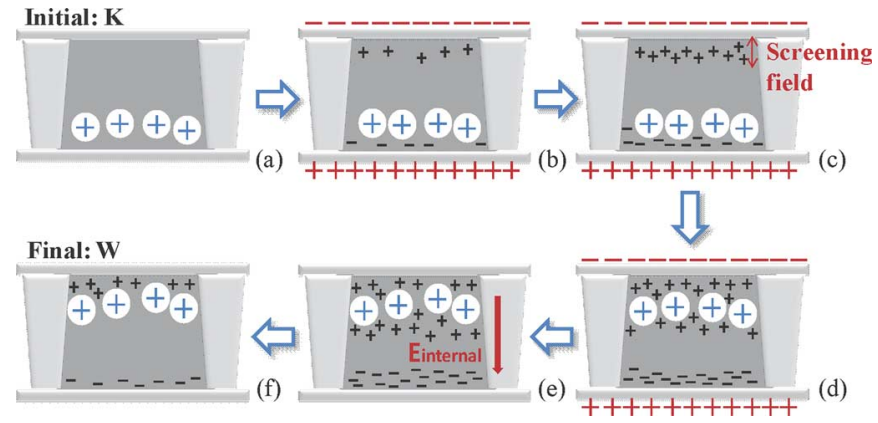

Fig. 4. Schema of charge movements inside a microcup EPD under application of an external electric field. The red signs outside of the microcup represent the applied voltage. The white circles and the black \pm signs represent dispersed particles and ions, respectively.

state current (stage II), and a discharge current (stage III), as shown in Fig. 3.

When an external field of a $300 \mathrm{~ms}, 30 \mathrm{~V}$ pulse voltage was applied, the transient current (stage I) responded instantly, whereas the optical response remained almost unchanged. This indicates that the transient current peak is mostly dominated by micelles and surfactant dissociation instead of by dispersed particles, as shown in Fig. 4(b). With a continuously applied external electric field, the ions and other charged species accumulated at the electrode, and a space charge region was formed, as shown in Fig. 4(c). This region did not contribute to the current; also, this region screened out the applied voltage, which resulted in a decrease in transient current [19].

At stage II, a bump (maximum current amplitude in stage II) appeared after the wake-up time in the intermediate-state current accompanying the steepest optical response. This indicated that dispersed particles had moved to the opposite electrode, as shown in Fig. 4(d). Consequently, the dominant contributor of the current bump in stage II was the dispersed particles.

After releasing the applied voltage, the optical response did not drop, but a reverse current peak appeared at the beginning of stage III. This implies that the induced internal electric field inside the microcup pushed the lighter dissociated ions and micelles away from the electrodes, whereas the heavier charged particles remained in the same position within the induced electric field, as shown in Fig. 4(e) and (f).

In the following section, these assumptions are tested and verified for both the optical and current responses, using different waveforms with the same current trends.

\section{Current Bump With Various Pulse Widths}

Four different pulse widths $(100,150,200$, and $300 \mathrm{~ms})$ of a $30 \mathrm{~V}$ applied voltage were designed and applied to a microcup EPD. The relationships between optical response and current behavior, including current bumps with different pulse widths, are shown in Fig. 5; the solid and dashed lines represent optical response (OR) and current response, respectively. The experimental results [Fig. 5(a)] illustrated the following.

1) Different pulse widths had the same wake-up time (approximately $90 \mathrm{~ms}$ ). This shows that the charges received identical strength during the preceding $100 \mathrm{~ms}$ of all four applied widths. 

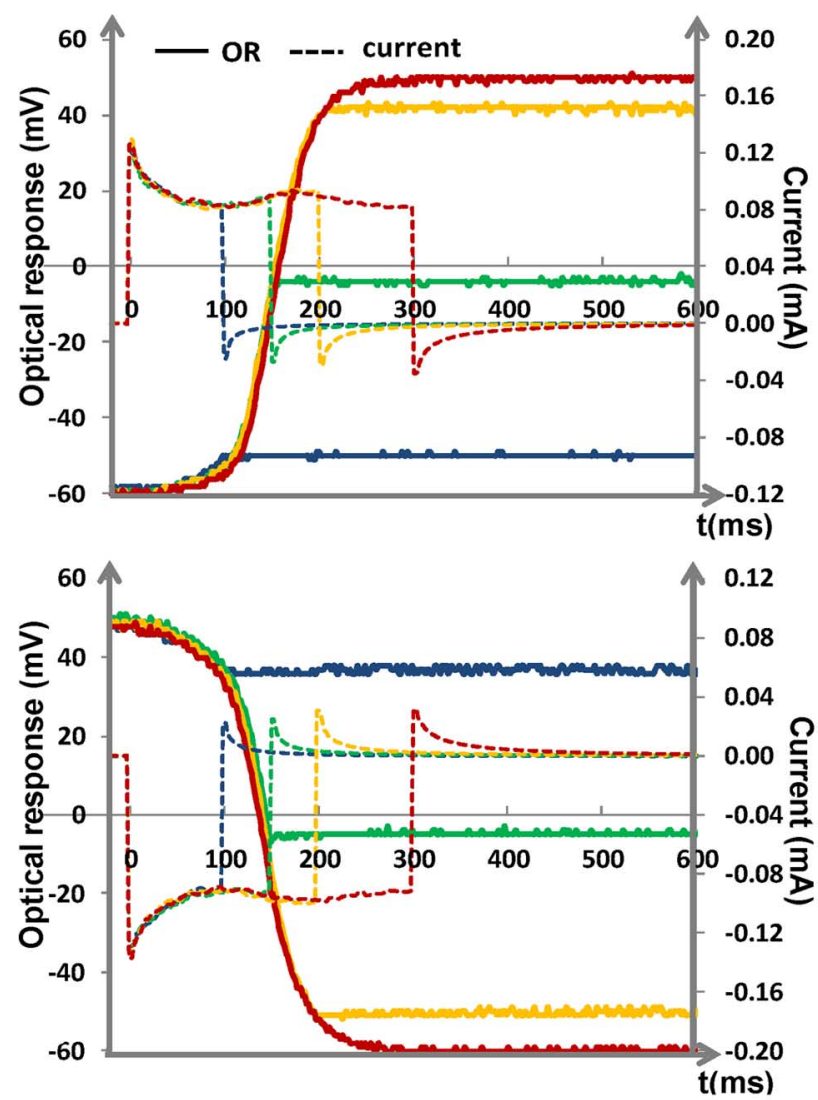

(a)

Fig. 5. Current and optical responses of a microcup EPD modulated by four different pulse widths $(100 \mathrm{~ms}, 150 \mathrm{~ms}, 200 \mathrm{~ms}$, and $300 \mathrm{~ms})$. The voltages applied were (a) $30 \mathrm{~V}$ (initial in the dark state) and (b) $-30 \mathrm{~V}$ (initial in the white state). The solid and dashed lines represent the optical response (OR) and current response, respectively.

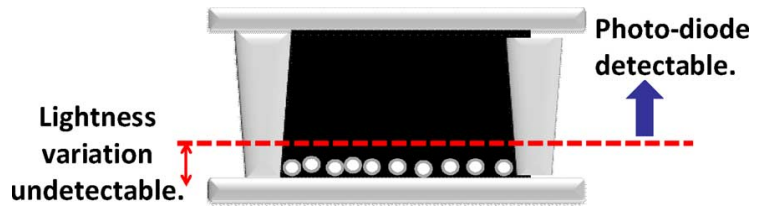

Fig. 6. Explanation of lightness detection.

2) Current bumps with different pulse widths were fixed at the same moment (approximately $180 \mathrm{~ms}$ ). Moreover, the current bump always occurred after the wake-up time, with a dramatic increase in optical response. That is, the current bump came from the movement of dispersed particles, as assumed in the previous section. Therefore, the optical response curves for the $100 \mathrm{~ms}$ (blue curve) and $150 \mathrm{~ms}$ (green curve) pulse widths did not reach an extreme white state because the pulse widths of the applied voltages were not long enough to completely drive particles out. Thus, the bump did not happen in the cases of the $100 \mathrm{~ms}$ (blue curve) and $150 \mathrm{~ms}$ (green curve) pulse widths.

3) When a pulse width was longer, surfactant dissociation rose accordingly and resulted in a higher discharge peak current, as shown in stage III. The experimental results indicate that both the transient current (stage I) and discharge current peaks (stage III) were generated by micelles or ions due to the retained optical response. The longer the pulse
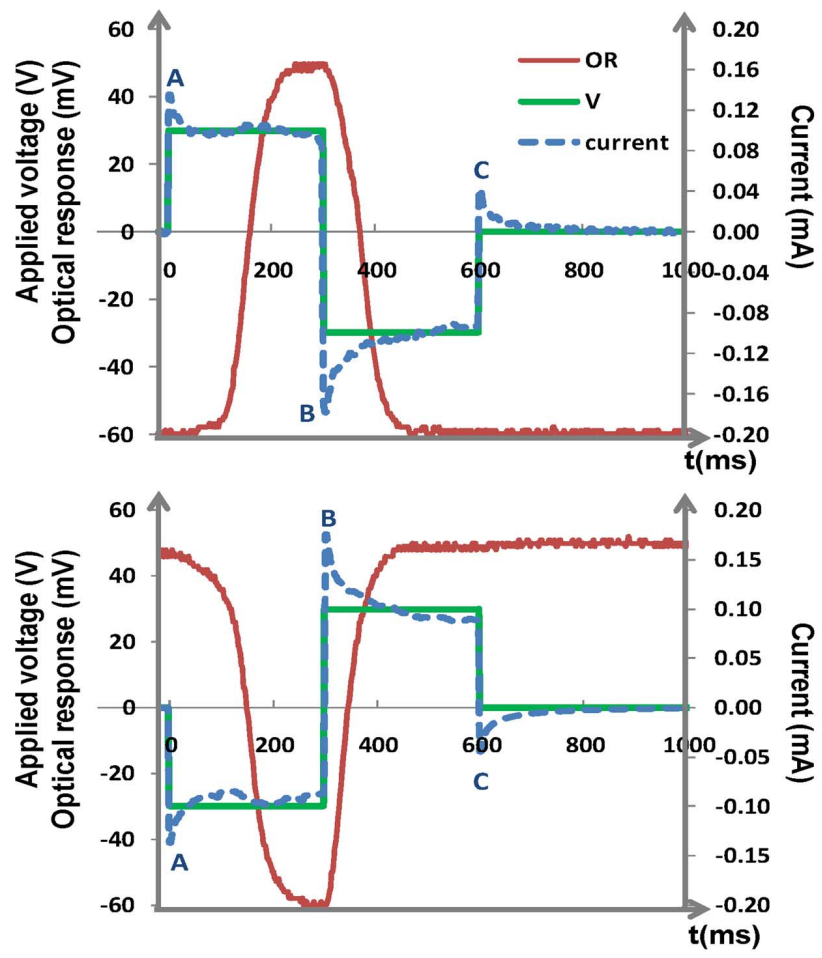

(a)

Fig. 7. Antisymmetric waveforms for (a) $300 \mathrm{~ms}, 30 \mathrm{~V}$ plus $300 \mathrm{~ms},-30 \mathrm{~V}$. (b) $300 \mathrm{~ms},-30 \mathrm{~V}$ plus $300 \mathrm{~ms}, 30 \mathrm{~V}$. The red solid curve is the optical response (OR), the green solid square is the applied voltage (V), and the blue dashed curve is the current response.

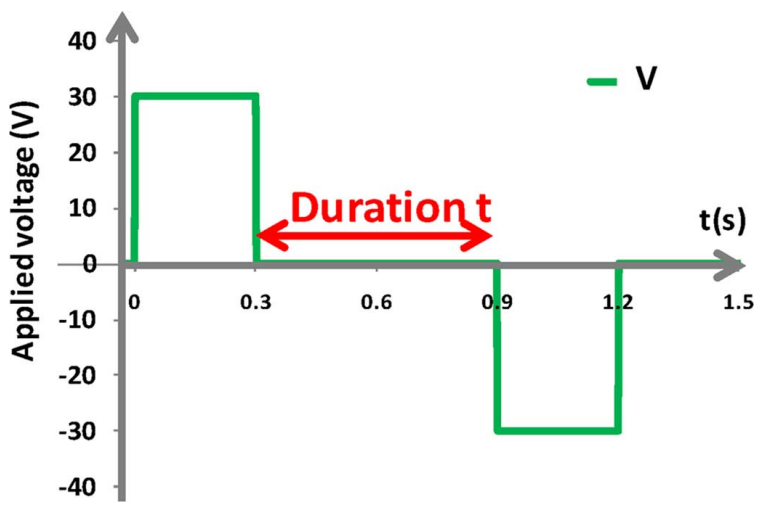

Fig. 8. An antisymmetric waveform with sequential pulses of $300 \mathrm{~ms} / 30 \mathrm{~V}$, a duration $t / 0 \mathrm{~V}$, and $300 \mathrm{~ms} /-30 \mathrm{~V}$.

width, the greater the amount of surfactant molecules dissociated, and the larger the internal electric field induced. Thus, the discharge current peak for the $300 \mathrm{~ms}$ pulse width was larger than the peaks for $200 \mathrm{~ms}, 150 \mathrm{~ms}$, and $100 \mathrm{~ms}$. Furthermore, the amplitude of the discharge current peak was less than half of the transient-current peak. This indicates that most of the ions and micelles remained near the electrode and helped to maintain EPD bistability, while some of the ions and micelles were pushed away and were recombined by an internal-induced electric field.

The reverse voltage experiment provided similar experimental results, as shown in Fig. 5(b). The major difference from the opposite magnitude experiments was that the wake-up times of the white state to the dark state were approximately 


\section{Optical response $(\mathrm{mV})$}

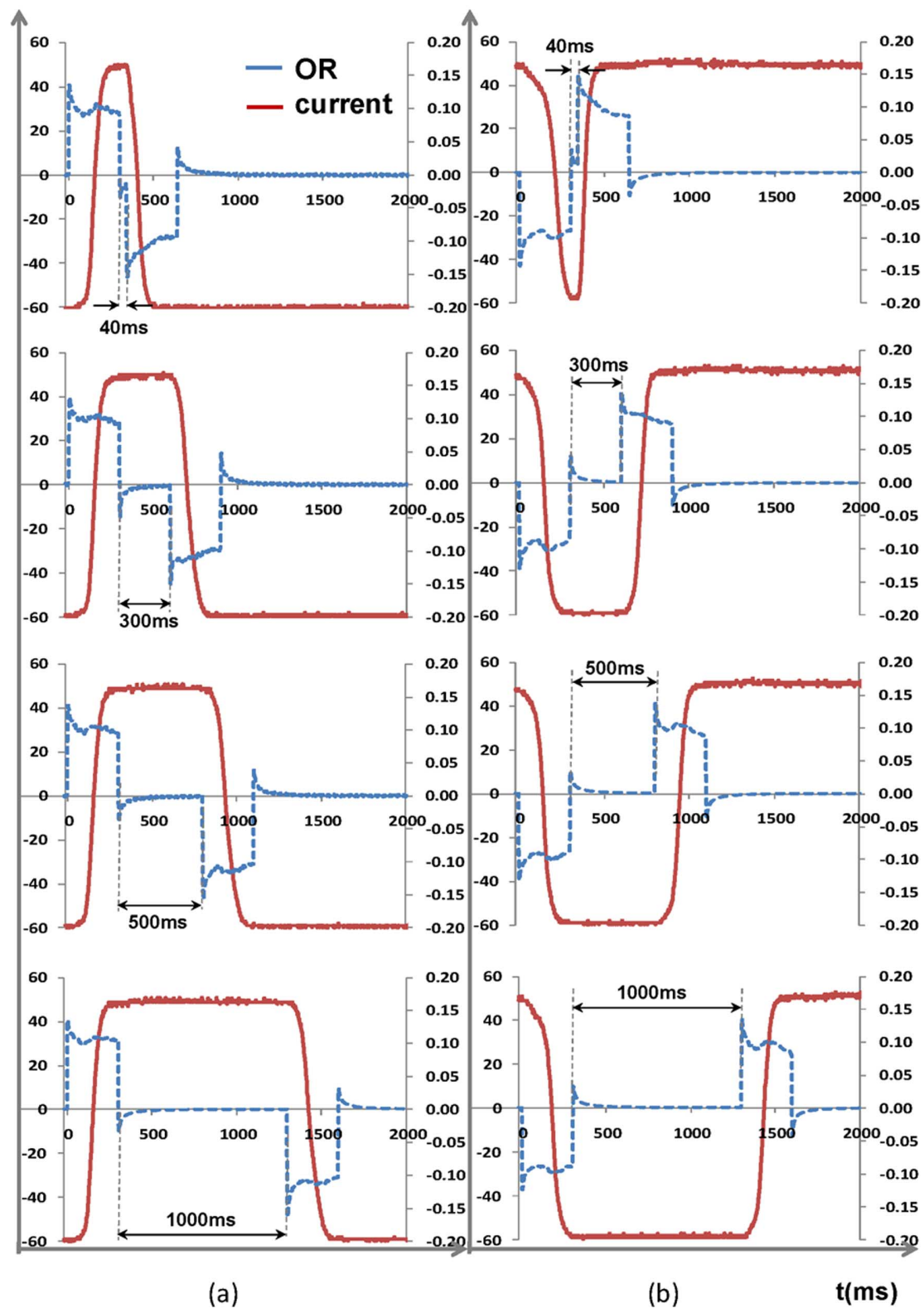

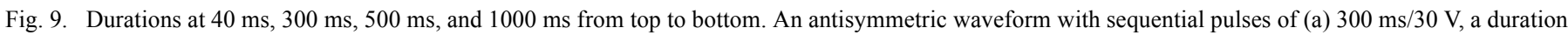
$\mathrm{t} / 0 \mathrm{~V}$, and $300 \mathrm{~ms} /-30 \mathrm{~V}$. (b) $300 \mathrm{~ms} /-30 \mathrm{~V}$, a duration $\mathrm{t} / 0 \mathrm{~V}$, and then $300 \mathrm{~ms} / 30 \mathrm{~V}$.

$60 \mathrm{~ms}, 30 \mathrm{~ms}$ shorter than the dark state to white state transition shown in Fig. 5(a). This might result from the absorption of light from the dark suspension. In the white state, the white particles are close to the top electrode, and lightness variation is more easily perceived when particles move to the opposite electrode. In contrast, in the dark state, dispersed particles are closer to the bottom electrode. Because the suspension is dark, the lightness might be undetectable until the dispersed particles move toward the top electrode and reach a threshold distance along the z-axis, as shown in Fig. 6. Therefore, the transition from white to dark state was shorter in response to the applied voltage. In other words, a shorter wake-up time was needed. Nevertheless, the largest change in the optical response occurred at the bump position.
The single-pulse experiment was critical for understanding the motion of charge materials inside a microcup EPD. However, the driving waveforms were generally combined by a series of pulses. In the following section, the optical and current responses are further studied using various driving waveforms.

\section{Current Bump With Antisymmetric Waveforms}

To further understand the correlation between bump position and optical response, a series of antisymmetric voltage waveforms were designed. An interesting phenomenon was observed in this experiment: there was no current bump when applying an opposite electric field (the second pulse), as shown in both Fig. 7(a) and (b). The current response results are compared with a single pulse voltage applied to an EPD, as shown in Fig. 3 . 
The transient peak current (peak A) at the first pulse and the discharge peak current (peak C) at the second phase of an antisymmetric waveform have almost the same current magnitude as that obtained by applying a single pulse voltage $(0.14 \mathrm{~mA}$ and $0.04 \mathrm{~mA}$, respectively), as shown in Fig. 3. These results verify that the transient and discharge currents are mostly produced by surfactant disassociation. The current magnitude would be the same regardless of the applied voltages, as discussed in part ii of Section C. However, the current $(0.18 \mathrm{~mA})$ of peak B was $28 \%$ larger than the first transient current of peak A $(0.14 \mathrm{~mA})$. Peak B was almost equal to the sum of transient peak $A$ and the bump in magnitude. Consequently, we speculated that peak B represents the superposition of currents contributed from both dispersed particles and ions.

For a single pulse, as mentioned in Section B, the dispersed particles and ions moved to the negatively charged electrode when a voltage was applied. After releasing the voltage, ions were pushed away from the electrode by a repulsive force from an induced internal electric field. After the relaxation time, as more ions left the electrode, spaces around the particles formed, i.e., particles felt less repulsive force from neighboring charges. In the antisymmetric waveform, however, there was no removal voltage in the second phase; a reverse voltage was observed instead. At this moment, the electric force from the induced internal and the applied external electric fields were in the same direction. These two electric forces accelerated the charged materials, just as hurried people might push each other while leaving a musical concert. Therefore, the positive dispersed particles were pushed and instantly moved to the new negative electrode by the surrounding positive ions when the applied voltage was reversed. This explained the higher current amplitude of peak B compared to that of peak $\mathrm{A}$, and it implied that a bump can hide inside peak B, as we speculated in the last paragraph.

If this speculation is true, the current bump will emerge again when there is enough time for ion relaxation. A new waveform was consequently designed to provide increased ion relaxation, as described in the next section.

\section{E. Antisymmetric Waveforms With an in-Between Inserted Duration}

To verify that a bump is buried in peak B and that the higher peak B current amplitude comes from the sum of a discharge current of the first phase (lack of relaxation time) and a transient current in the second phase, antisymmetric waveforms separated by various durations were designed, as shown in Fig. 8. If the duration provides charges with enough relaxation time, the bump in the second phase is expected to appear again.

Four durations were tested, including $t=40 \mathrm{~ms}, 300 \mathrm{~ms}$, $500 \mathrm{~ms}$, and $1000 \mathrm{~ms}$. Fig. 9(a) shows that the current bump emerges after $500 \mathrm{~ms}$. The wake-up time shows the same trend in Table I. Below $500 \mathrm{~ms}$, the wake-up time grew longer as duration increased; it reached a stable value after $500 \mathrm{~ms}$. This implies that shorter durations lead to a shorter transition time with less ion relaxation. The reverse voltage experiment gave similar results, as shown in Fig. 9(b). This indicates the appearance of the bump is related not just to the degree of ion relaxation but
TABLE I

WAKE-UP TIME (WUT) FOR AN ANTISYMMETRIC WAVEFORM WITH Duration $t$ Inserted Between Two Phases

\begin{tabular}{|c|c|c|c|c|c|c|}
\hline & Duration $\mathrm{t}(\mathrm{ms})$ & 0 & 40 & 300 & 500 & 1000 \\
\hline $\begin{array}{r}\text { WUT } \\
(\mathrm{ms})\end{array}$ & $\mathrm{W} \rightarrow \mathrm{K}$ & 4 & 4 & 44 & 64 & 64 \\
\hline
\end{tabular}

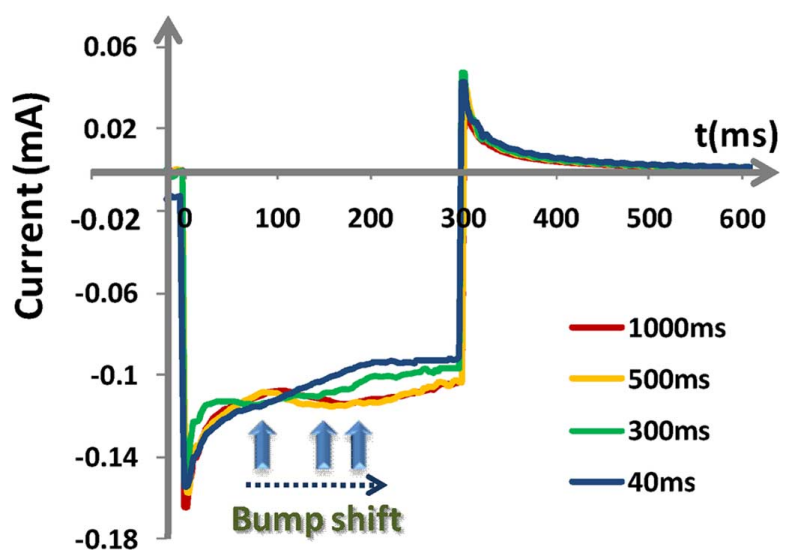

Fig. 10. Four overlapping current pulses from Fig. 8(a) (the four experimental time axes are shifted for a convenient comparison).

also the length of the wake-up time and transition time. Moreover, the wake-up time was almost zero with a zero pulse duration (antisymmetric waveform as shown in Fig. 7); this could provide a way to shorten the driving time.

Four pulses of the second phase in Fig. 9(a) were shifted and grouped together, as shown in Fig. 10. The current bump gradually appeared when duration time was increased. This suggests that the bump was buried in the second pulse. Additionally, when the duration was long enough $(500 \mathrm{~ms}$ in this experiment) for ion relaxation, the current bump emerged. This also suggests that, if the bump were to appear earlier due to the use of a different voltage waveform, the optical response time could be shortened.

\section{APPLICATION}

The current bump represented the moment of lightness transition to an extreme state, as mentioned in Section C. The antisymmetric waveform reduced the transition time in Section D. The optical response time was reduced if the bump appeared earlier due to the use of different reverse voltage waveforms, as shown in Section E. Combining these three experimental results, a waveform for shortening the transition time was designed, as shown in Fig. 11. A short reverse pulse was inserted before the original one, which reduced the optical response time of the extreme state transition. The reverse pulse should not change the original optical state; the optical response remained the same amplitude with the reverse pulse, so the waveform can be shortened without changing the lightness.

A comparison of the $300 \mathrm{~ms}$ pulse and the proposed waveform (a $20 \mathrm{~ms}$ negative pulse width inserted before the $300 \mathrm{~ms}$ positive pulse width) is shown in Fig. 12(a). The experimental results showed the designed waveform reduces the transition 


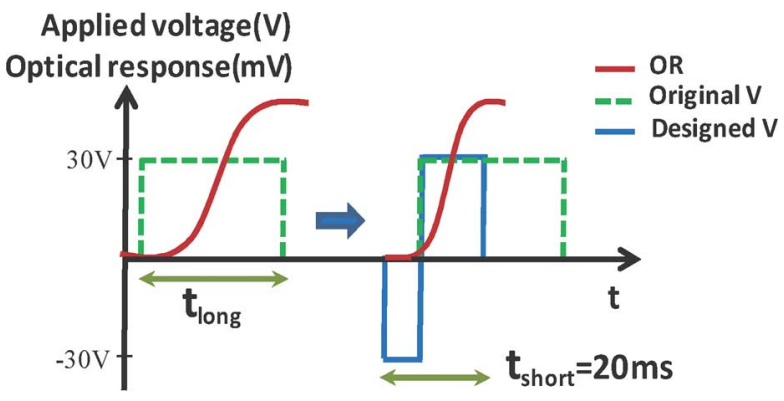

Fig. 11. Schematic of the proposed driving waveform to reduce transition time.
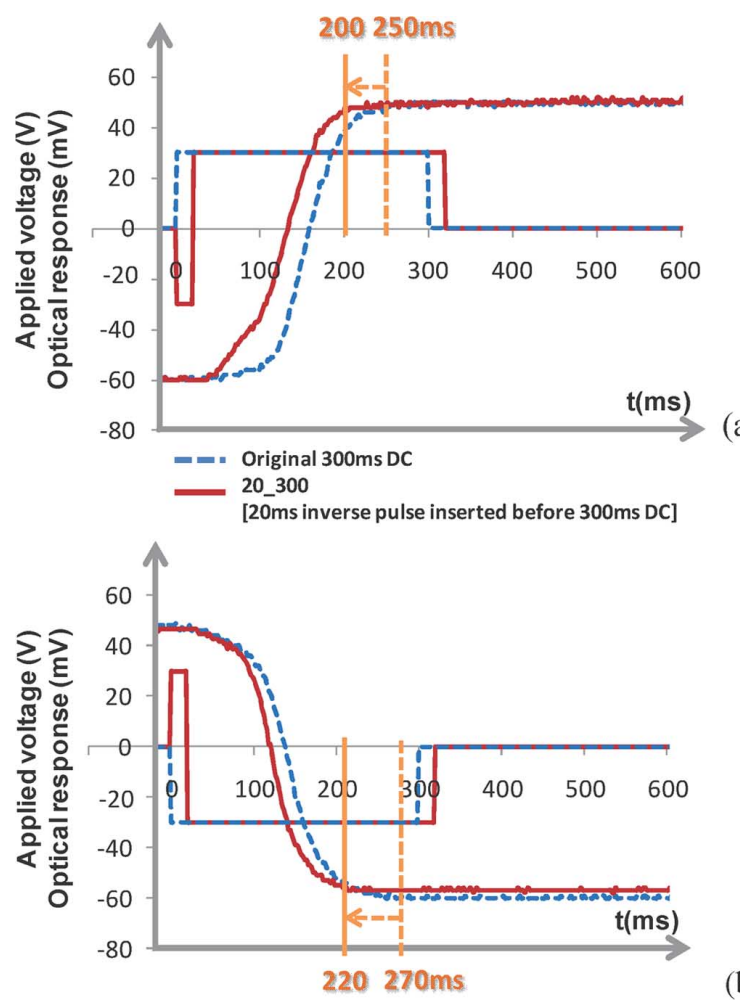

(b)

Fig. 12. The blue dashed lines represent the applied voltage and optical response of a $30 \mathrm{~V}, 300 \mathrm{~ms}$ pulse. The red lines represent the applied voltage and optical response of a short $20 \mathrm{~ms}$ inverse pulse inserted in front of a $300 \mathrm{~ms}$ pulse. Driving from (a) dark to white state and (b) white to dark state.

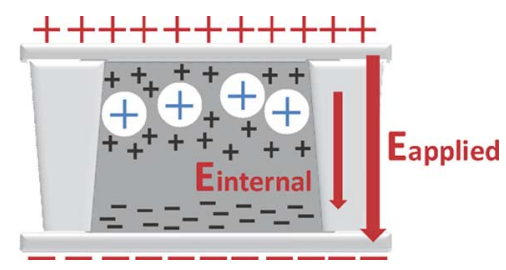

Fig. 13. Illustration of the electric fields at the $\mp$ applied voltage transform stage.

time by $50 \mathrm{~ms}$. Additionally, the total driving time was reduced from $250 \mathrm{~ms}$ to $200 \mathrm{~ms}$ (a $20 \%$ improvement). The inverse experiment gave similar results, as shown in Fig. 12(b). The designed waveform successfully reduced the transition time by $20 \%$, which means that in experiment part D, the speed of the charged species was greater due to the addition of an internal electric field and an applied field, as shown in Fig. 13.

\section{CONCLUSION}

The physical mechanism of the motion of charged species inside a microcup EPD was established and verified. Current responses, as well as optical responses, were observed to decipher the transition mechanism. The experiments showed that the presence or absence of a current bump did not depend on the widths of the applied voltages (as shown in the experiments using a single pulse voltage) but instead depended on the duration between the previous and present pulse (as shown in the experiment using an asymmetric pulse voltage). Moreover, the timing of the current bump also indicated the degree of ion relaxation inside the cell. We also observed that a current bump was caused by the rapid motion of dispersed particles. Therefore, the appearance of a current bump can serve as an indicator of optical response. For certain applications, the width of the total waveform could be shortened by finding ways to make the bump appear earlier. To this end, we propose an antisymmetric pulse voltage waveform that would reduce the transition time by $20 \%$.

\section{ACKNOWLEDGMENT}

The authors would like to express their appreciation to their classmates S.-H. Yang, P.-C. Hsu, C.-Y. Hsu, and L.-M. Yang. Furthermore, they are grateful for assistance from their collaborators, M.-J. Chang, J.-C. Huang, and Y.-J. Hsieh, at SiPix Corporation, New Taipei City, Taiwan.

\section{REFERENCES}

[1] F. C. Lin, Y.-P. Huang, L.-Y. Liao, C.-Y. Laio, H.-P. P. D. Shieh, T.-M. Wang, and S.-C. Yeh, "Dynamic backlight gamma on high dynamic range LCD TVs," J. Display Technol., vol. 4, no. 2, pp. 139-152, Jun. 2008.

[2] F. C. Lin et al., "Color breakup suppression and low power consumption by stencil-FSC method in field-sequential LCDs," J. Soc. Inf. Display, vol. 17, no. 3, pp. 221-228, 2009.

[3] Y. P. Huang, F. C. Lin, and H.-P. Shieh, "Eco-displays: The color LCD's without color filters and polarizers," J. Display Technol., vol. 7, no. 12, pp. 630-632, Dec. 2011.

[4] R. C. Liang et al., "Microcup displays: Electronic paper by manufacturing process," J. Soc. Inf. Display, vol. 11, no. 4, pp. 621-628, 2003.

[5] D. D. Mysko and J. C. Berg, "Mechanisms influencing the stability of a nonaqueous phosphor dispersion," Ind. Eng. Chem. Res., vol. 32, pp. $854-858,1993$.

[6] R. M. Webber, "Image stability in active-matrix microencapsulated electrophoretic displays," in SID Dig., 2002, pp. 126-129.

[7] W. C. Kao, J.-A. Ye, M.-I Chu, and C.-Y. Su, "Image quality improvement for electrophoretic displays by combining contrast enhancement and halftoning techniques," IEEE Trans. Consumer Electron., vol. 55, no. 1, pp. 15-19, Feb. 2009.

[8] T. Bert and H. D. Smet, "Dielectrophoresis in electronic paper," Displays, vol. 24, pp. 223-230, 2003.

[9] Y. Sakka and T. Uchikoshi, "Forming and microstructure control of ceramics by electrophoretic deposition," KONA: Powder and Particle $J .$, pp. 74-87, 2010.

[10] M. D. Croucher and M. L. Hair, "Some physical properties of electrophoretic display materials," Ind. Eng. Chem. Prod. Res., vol. 20, no. 2, pp. 324-329, 1981.

[11] T. Bert and H. De Smet, "How to introduce a threshold in EPIDs," in IDRC, 2005. 
[12] R. Pool and P. G. Bolhuis, "The influence of micelle formation on the stability of colloid surfactant mixtures," Phys. Chem. Chem. Phys., vol. 12, pp. 14789-14797, 2010.

[13] L. Besra and M. Liu, "A review on fundamentals and applications of electrophoretic deposition (EPD)," Progr. Mater. Sci., vol. 52, pp. $1-61,2007$.

[14] T. Bert et al., "Steady state current in EPIDs," Displays, vol. 27, pp. $35-38,2006$.

[15] T. Bert et al., "Transient current properties in electronic paper," in $I D W$, 2004, pp. 1749-1752.

[16] T. Bert and H. De Smet, "The microscopic physics of electronic paper revealed," Displays, vol. 24, pp. 103-110, 2003.

[17] G. S. Roberts et al., "Electrostatic charging of nonpolar colloids by reverse micelles," Langmuir, vol. 24, pp. 6530-6541, 2008.

[18] I. Chen et al., "Particle charge, mobility, and zeta potential in nonpolar colloids," Langmuir, vol. 13, pp. 5036-5040, 1997.

[19] Y. Jeon et al., "Understanding electrophoretic displays: Transient current characteristics of dispersed charges in a non-polar medium," in IDW, 2010.

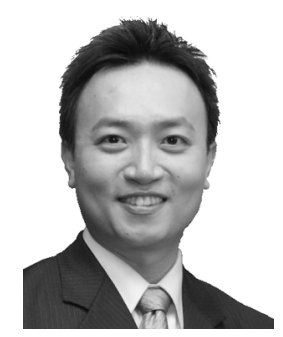

F.-C. Lin received the B.S. and M.S. degrees from the Department of Physics, National Cheng Kung University, Taiwan, in 2000 and 2002, respectively, and the Ph.D. degree from the Institute of Electro-Optical Engineering, National Chiao Tung University, Hsinchu, Taiwan, in 2009.

During 2009/9-2010/8, he was a visiting scientist in Video \& Image Processing at Philips Research, the Netherlands. Currently he is an assistant research fellow at Display Institute, National Chiao Tung University and researching on e-paper, brain-computer interfaces (BCIs), and developing Eco-display systems.

In his Ph.D. career, Dr. Lin won the 2009 JSID Outstanding Student Paper of the Year Award.

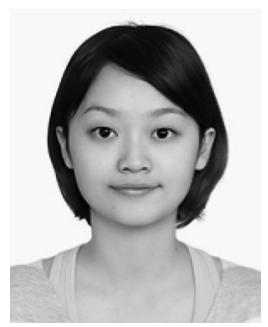

S.-I Wu received the B.S. and M.S. degrees from the Department of Electrophysics and Institute of Electro-Optical Engineering, National Chiao Tung University (NCTU), Hsinchu, Taiwan, in 2009 and 2011, respectively.

Currently she is a patent engineer at Jianq Chyun Intellectual Property Office, Taipei, Taiwan, and responsible for the field of optoelectronic and optics, including displays (flat panel displays, electronic-papers, stereo displays), thin-film transistors (TFTs), solar cells, light-emitting diodes (LEDs), organic light-emitting diodes (OLEDs) and light guide plates, etc.

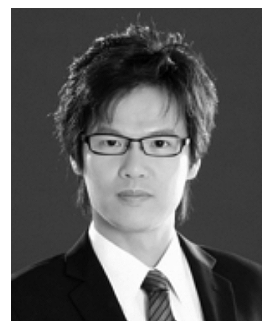

P.-R. Yang received the B.S. degree from the Department of Material Science and Engineering, National Chiao Tung University (NCTU), Taiwan, in 2002, the M.S. degree from National Tsing Hua University, Taiwan, in 2004, and the Ph.D. degree from the Institute of Electro-Optical Engineering from NCTU, Taiwan, in 2008.

During his time studying Ph.D. degree, he received the scholarships to study fast-switching LCD at University of Oxford, U.K., in 2007, and Tohoku University, Japan, in 2008 , as a doctoral researcher. Also, he received the scholarship from Applied Materials Taiwan to visit Applied Materials' sponsored research centers in University of California, Berkeley, CA, USA, and Stanford University, Stanford, CA, USA, in 2005. Since 2009, he has been with SiPix Technology Inc., New Taipei City, Taiwan, working on the fundamental mechanism of electrophoretic display at the research center located in Fremont, CA, USA, and product development at the manufacturing site located in Taiwan. He is currently an R\&D manager in SiPix responsible for new product development
Dr. Huang was awarded the SID2001 Best Student Paper Award, SID2004 distinguished student paper award, 2005 Golden Thesis Award of Acer Foundation, and 2005 AUO Bravo Award.
Yi-Pai Huang the B.S. degree from National degree from the Institute of Opto-Electronic EngiHsinchu, Taiwan.

partment of Photonics \& Display Institute, Nationa internship student. He had successfully developed "advanced-MVA LCD the next generation products of AUO in 2005. His current research interests are advanced display systems (high-dynamic-range LCD and field-sequential-color

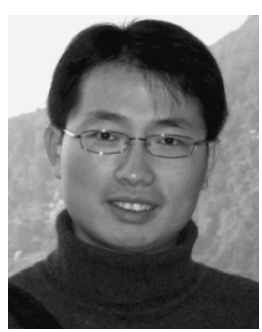$$
\text { (1) }
$$ 